\title{
On Bivariate Exponentiated Extended Weibull Family of Distributions
}

\author{
Rasool Roozegar*1 and Ali Akbar Jafari ${ }^{2}$ \\ ${ }^{1,2}$ Department of Statistics, Yazd University, Yazd, Iran
}

\begin{abstract}
In this paper, we introduce a new class of bivariate distributions called the bivariate exponentiated extended Weibull distributions. The model introduced here is of Marshall-Olkin type. This new class of bivariate distributions contains several bivariate lifetime models. Some mathematical properties of the new class of distributions are studied. We provide the joint and conditional density functions, the joint cumulative distribution function and the joint survival function. Special bivariate distributions are investigated in some detail. The maximum likelihood estimators are obtained using the EM algorithm. We illustrate the usefulness of the new class by means of application to two real data sets.
\end{abstract}

Keywords: Bivariate exponentiated extended Weibull distribution, Joint probability density function, EM-algorithm, Maximum likelihood estimation. 


\section{Introduction}

The Weibull distribution has assumed a prominent position as statistical model for data from reliability, engineering and biological studies (McCool, 2012). The Weibull distribution is a reasonable choice due to its negatively and positively skewed density shapes. However, this distribution is not a good model for describing phenomenon with non-monotone failure rates, which can be found on data from applications in reliability studies. Thus, extended forms of the Weibull model have been sought in many applied areas. As a solution for this issue, the inclusion of additional parameters to a well-defined distribution has been indicated as a good methodology for providing more flexible new classes of distributions.

The class of extended Weibull (EW) distributions pioneered by Gurvich et al. (1997) has achieved a prominent position in lifetime models. Its cumulative distribution function (cdf) is given by

$$
G(x ; \lambda, \xi)=1-e^{-\lambda H(x ; \xi)}, \quad x>0, \quad \lambda>0,
$$

where $H(x ; \xi)$ is a non-negative monotonically increasing function which depends on the parameter vector $\xi$. The corresponding probability density function (pdf) is given by

$$
\mathrm{g}(x ; \lambda, \boldsymbol{\xi})=\lambda h(x ; \xi) e^{-\lambda H(x ; \xi)}, \quad x>0, \quad \lambda>0,
$$

where $h(x ; \xi)$ is the derivative of $H(x ; \xi)$. We emphasize that several distributions could be expressed in the form (1). Table 1 summarizes several of these models. Further, we refer the reader to Nadarajah and Kotz (2005) and Pham and Lai (2007).

In recent years, many authors worked on this class of distributions such as the beta extended Weibull family by Cordeiro et al. (2012), the extended Weibull power series distributions by Silva et al. (2013), the complementary extended Weibull power series class of distributions by Cordeiro and Silva (2014), the Marshall-Olkin extended Weibull family of distributions by Santos-Neto et al. (2014) and the exponentiated extended Weibull power series class of distributions by Tahmasebi and Jafari (2015).

The aim of this paper is to introduce a new bivariate exponentiated extended Weibull (BEEW) family of distributions, whose marginals are exponentiated extended Weibull (EEW) distributions. It is obtained using a method similar to that used to obtain Marshall-Olkin bivariate exponential model (Marshall and Olkin, 1967). The proposed BEEW class of distributions is constructed from three independent EEW distributions using a maximization process. Creating a bivariate distribution with given marginals using this technique is nothing new.
The joint cdf can be expressed as a mixture of an absolutely continuous cdf and a singular cdf. The joint pdf of the BEEW distributions can take different shapes and the cdf can be expressed in a compact form. The joint cdf, the joint pdf and the joint survival function (sf) are in closed forms, which make it convenient to use in practice. The new class of bivariate distributions contains as special models the bivariate generalized exponential (Kundu and Gupta, 2009), bivariate generalized linear failure rate (Sarhan et al., 2011), bivariate generalized Gompertz (El-Sherpieny et al., 2013), bivariate exponentiated generalized Weibull-Gompertz (El-Bassioun et al., 2015), bivariate exponentiated modified Weibull extension (El-Gohary and El-Morshedy, 2015) distributions. This class defines at least $46(2 \times 23)$ bivariate sub-models as special cases.

The usual maximum likelihood estimators can be obtained by solving non-linear equations in at least five unknowns directly, which is not a trivial issue. To avoid difficult computation we treat this problem as a missing value problem and use the EM algorithm, which can be implemented more conveniently than the direct maximization process. Another advantage of the EM algorithm is that it can be used to obtain the observed Fisher information matrix, which is helpful for constructing the asymptotic confidence intervals for the parameters. Alternatively, it is possible to obtain approximate maximum likelihood estimators by estimating the marginals first and then estimating the dependence parameter through a copula function, as suggested by (Joe, 1997, Chapter 10), which has the same rate of convergence as the maximum likelihood estimators. This is computationally less involved compared to the MLE calculations. This approach is not pursued here. Although in this paper we mainly discuss the BEEW, many of our results can be easily extended to the multivariate case.

The main reasons for introducing this new class of bivariate distributions are: (i) This class of distributions is an important model that can be used in a variety of problems in modeling bivariate lifetime data. (ii) It provides a reasonable parametric fit to skewed bivariate data that cannot be properly fitted by other distributions. (iii) The joint cdf and joint pdf should preferably have a closed form representation; at least numerical evaluation should be possible. (v) This class contains several special bivariate models because of the general class of Weibull distributions and the fact that the current generalization provides means of its bivariate continuous extension to still more complex situations; therefore it can be applied in modeling bivariate lifetime data.

The rest of the paper is organized as follows. We define the EEW and the BEEW class of distributions in Section 2. Different properties of this family are discussed in this section. The special cases of the BEEW model are considered in Section 3. The EM algorithm 
Table 1: Special cases of EW distributions and corresponding $H(x ; \xi)$ function

\begin{tabular}{l|c|c|l|c|l}
\hline Distribution & Support & $H(x ; \xi)$ & $\lambda$ & $\xi$ & \multicolumn{1}{c}{ Reference } \\
\hline Exponential & $x \geq 0$ & $x$ & $\lambda$ & $\varnothing$ & Johnson et al. (1995) \\
\hline Pareto & $x=k$ & $\log (x / k)$ & $\lambda$ & $k$ & Johnson et al. (1995) \\
\hline Gompertz & $x \geq 0$ & $c^{-1}[\exp (c x)-1]$ & $\lambda$ & $c$ & Gompertz (1825) \\
\hline Weibull & $x \geq 0$ & $x^{\gamma}$ & $\lambda$ & $\gamma$ & Fréchet (1927) \\
\hline Fréchet & $x \geq 0$ & $x^{-\gamma}$ & $\lambda$ & $\gamma$ & Fréchet (1927) \\
\hline Lomax & $x \geq 0$ & $\log (1+x)$ & $\lambda$ & $\varnothing$ & Lomax (1954) \\
\hline Weibull Kies & $0<\mu<x<\sigma$ & $(x-\mu)^{b_{1}} /(\sigma-x)^{b_{2}}$ & $\lambda$ & $\left(\mu, \sigma, b_{1}, b_{2}\right)$ & Kies (1958) \\
\hline Log-logistic & $x \geq 0$ & $\log \left(1+x^{c}\right)$ & $\lambda$ & $c$ & Fisk (1961) \\
\hline Linear failure rate & $x \geq 0$ & $a x+b x^{2} / 2$ & 1 & $(a, b)$ & Barlow (1968) \\
\hline Log-Weibull & $-\infty<x<\infty$ & $\exp [(x-\mu) / \sigma]$ & 1 & $(\mu, \sigma)$ & White (1969) \\
\hline Exponential power & $x \geq 0$ & $\exp \left((c x)^{a}-1\right)$ & 1 & $(a, c)$ & Smith and Bain (1975) \\
\hline Burr XII & $x \geq 0$ & $\log \left(1+x^{c}\right)$ & $\lambda$ & $c$ & Rodriguez (1977) \\
\hline Rayleigh & $x \geq 0$ & $x^{2}$ & $\lambda$ & $\varnothing$ & Rayleigh (1880) \\
\hline Phani & $0<\mu<x<\sigma$ & {$[(x-\mu) /(\sigma-x)]^{b}$} & $\lambda$ & $(\mu, \sigma, b)$ & Phani (1987) \\
\hline Additive Weibull & $x \geq 0$ & $\left(x / \beta_{1}\right)^{\alpha_{1}}+\left(x / \beta_{2}\right)^{\alpha_{2}}$ & 1 & $\left(\alpha_{1}, \alpha_{2}, \beta_{1}, \beta_{2}\right)$ & Xie and Lai (1995) \\
\hline Chen & $x \geq 0$ & $\exp \left(x^{b}-1\right)$ & $\lambda$ & $b$ & Chen (2000) \\
\hline Pham & $x \geq 0$ & $\left(a^{x}\right)^{\beta}-1$ & 1 & $(a, \beta)$ & Pham (2002) \\
\hline Weibull extension & $x \geq 0$ & $c\left[\exp (c x)^{b}-1\right]$ & $\lambda$ & $(\gamma, b, c)$ & Xie et al. (2002) \\
\hline Modified Weibull & $x \geq 0$ & $x^{\gamma} \exp (c x)$ & $\lambda$ & $(\gamma, c)$ & Lai et al. (2003) \\
\hline Traditional Wibull & $x \geq 0$ & $x^{d} \exp \left(c x^{a}-1\right)$ & $\lambda$ & $(a, b, c)$ & Nadarajah and Kotz (2005) \\
\hline Generalized Weibull power & $x \geq 0$ & {$\left[1+(x / a)^{b}\right]^{c}-1$} & 1 & $(a, b, c)$ & Nikulin and Haghighi (2006) \\
\hline Flexible Weibull extension & $x \geq 0$ & $\exp \left(\alpha_{1} x-\beta_{1} / x\right)$ & 1 & $\left(\alpha_{1}, \beta_{1}\right)$ & Bebbington et al. (2007) \\
\hline Almalki Additive Weibull & $x \geq 0$ & $a x^{\theta}+b x^{\gamma} e^{c x}$ & 1 & $(a, b, c, \theta, \gamma)$ & Almalki and Yuan (2013) \\
\hline
\end{tabular}

to compute the MLEs of the unknown parameters is provided in Section 4. The analysis of two real data sets are provided in Section 5. Finally, we conclude the paper in Section 6.

\section{The BEEW model}

In this section, we introduce the BEEW distributions using a method similar to that which was used by Marshall and Olkin (1967) to define the Marshall Olkin bivariate exponential (MOBE) distribution.

First, consider the univariate EEW class of distributions with cdf given by

$F_{\mathrm{EEW}}(x ; \alpha, \lambda, \xi)=\left(1-e^{-\lambda H(x ; \xi)}\right)^{\alpha}, \quad x>0, \alpha>0, \lambda>0$.

The corresponding pdf is

$f_{\mathrm{EEW}}(x ; \alpha, \lambda, \xi)=\alpha \lambda h(x ; \xi) e^{-\lambda H(x ; \xi)}\left(1-e^{-\lambda H(x ; \xi)}\right)^{\alpha-1}$

From now on a EEW class of distributions with the shape parameter $\alpha$, the scale parameter $\lambda$ and parameter vector $\xi$ will be denoted by $\operatorname{EEW}(\alpha, \lambda, \xi)$. Note that many well-known models could be expressed in the form (3), such as exponentiated Weibull (Mudholkar and Srivastava, 1993), generalized exponential (Gupta and Kundu, 1999), Weibull extension (Chen, 2000), gen- eralized Rayleigh (Surles and Padgett, 2001; Kundu and Raqab, 2005), modified Weibull extension (Xie et al., 2002), generalized modified Weibull (Carrasco et al. 2008) generalized linear failure rate (Sarhan and Kundu, 2009), generalized Gompertz (El-Gohary et al., 2013), and exponentiated modified Weibull extension (Sarhan and Apaloo, 2013) distributions.

When $\alpha$ is a positive integer, the EEW model can be interpreted as the lifetime distribution of a parallel system consisting of $\alpha$ independent and identical units whose lifetime follows the EEW distributions.

From now on unless otherwise mentioned, it is assumed that $\alpha_{1}>0 ; \alpha_{2}>0 ; \alpha_{3}>0$ and $\lambda>0$. Suppose $U_{1} \sim \operatorname{EEW}\left(\alpha_{1}, \lambda, \xi\right), U_{2} \sim \operatorname{EEW}\left(\alpha_{2}, \lambda, \xi\right)$ and $U_{3} \sim$ $\operatorname{EEW}\left(\alpha_{3}, \lambda, \xi\right)$ and they are mutually independent. Here " $\sim$ " means follows or has the distribution. Now define $X_{1}=\max \left\{U_{1}, U_{3}\right\}$ and $X_{2}=\max \left\{U_{2}, U_{3}\right\}$. Then, we say that the bivariate vector $\left(X_{1}, X_{2}\right)$ has a bivariate exponentiated extended Weibull distribution with the shape parameters $\alpha_{1}, \alpha_{2}$ and $\alpha_{3}$, the scale parameter $\lambda$ and parameter vector $\xi$. We will denote it by $\operatorname{BEEW}\left(\alpha_{1}, \alpha_{2}, \alpha_{3}, \lambda, \boldsymbol{\xi}\right)$. Before providing the joint $\mathrm{cdf}$ or pdf, we first mention how it may occur in practice.

According to Kundu and Gupta (2009), suppose a system has two components and it is assumed that each component has been maintained independently and also there is an overall maintenance. Due to component maintenance, suppose the lifetime of the individual com- 
ponent is increased by $U_{i}$ amount and because of the overall maintenance, the lifetime of each component is increased by $U_{3}$ amount. Therefore, the increased lifetimes of the two component are $X_{1}=\max \left\{U_{1}, U_{3}\right\}$ and $X_{2}=\max \left\{U_{2}, U_{3}\right\}$, respectively.

We now study the joint cdf of the bivariate random vector $\left(X_{1}, X_{2}\right)$ in the following theorem.

Theorem 2.1. If $\left(X_{1}, X_{2}\right) \sim \operatorname{BEEW}\left(\alpha_{1}, \alpha_{2}, \alpha_{3}, \lambda, \xi\right)$, then the joint cdf of $\left(X_{1}, X_{2}\right)$ for $x_{1}>0, x_{2}>0$, is

$$
\begin{aligned}
F_{\mathrm{BEEW}}\left(x_{1}, x_{2}\right)= & \left(1-e^{-\lambda H\left(x_{1} ; \xi\right)}\right)^{\alpha_{1}}\left(1-e^{-\lambda H\left(x_{2} ; \xi\right)}\right)^{\alpha_{2}} \\
& \times\left(1-e^{-\lambda H(z ; \xi)}\right)^{\alpha_{3}}
\end{aligned}
$$

where $z=\min \left\{x_{1}, x_{2}\right\}$.

Proof. Since the joint cdf of the random variables $X_{1}$ and $X_{2}$ is defined as

$$
\begin{aligned}
F_{\text {BEEW }}\left(x_{1}, x_{2}\right)= & P\left(X_{1} \leq x_{1}, X_{2} \leq x_{2}\right) \\
= & P\left(\max \left\{U_{1}, U_{3}\right\} \leq x_{1},\right. \\
& \left.\max \left\{U_{2}, U_{3}\right\} \leq x_{2}\right) \\
= & P\left(U_{1} \leq x_{1}, U_{2} \leq x_{2}, U_{3} \leq\right. \\
& \left.\min \left(x_{1}, x_{2}\right)\right) .
\end{aligned}
$$

As the random variables $U_{i},(i=1,2,3)$ are mutually independent, we directly obtain

$$
\begin{gathered}
F_{\mathrm{BEEW}}\left(x_{1}, x_{2} ; \alpha_{1}, \alpha_{2}, \alpha_{3}, \lambda, \boldsymbol{\xi}\right)=F_{\mathrm{EEW}}\left(x_{1} ; \alpha_{1}, \lambda, \boldsymbol{\xi}\right) \\
\quad \times F_{\mathrm{EEW}}\left(x_{2} ; \alpha_{2}, \lambda, \boldsymbol{\xi}\right) F_{\mathrm{EEW}}\left(z ; \alpha_{3}, \lambda, \boldsymbol{\xi}\right)
\end{gathered}
$$

Substituting from 3 into 6, we obtain 5, which completes the proof of the theorem.

Corollary 1. The joint $c d f$ the $\operatorname{BEEW}\left(\alpha_{1}, \alpha_{2}, \alpha_{3}, \lambda, \xi\right)$ can also written as

$$
\begin{aligned}
F_{\text {BEEW }}\left(x_{1}, x_{2}\right) & =\left\{\begin{array}{lll}
b_{1} & \text { if } & x_{1} \leq x_{2} \\
b_{2} & \text { if } & x_{1}>x_{2}
\end{array}\right. \\
& =\left\{\begin{array}{lll}
c_{1} & \text { if } & x_{1}<x_{2} \\
c_{2} & \text { if } & x_{2}<x_{1} \\
c_{0} & \text { if } & x_{1}=x_{2}=x,
\end{array}\right.
\end{aligned}
$$

where

$$
\begin{aligned}
& b_{1}=\left(1-e^{-\lambda H\left(x_{1} ; \xi\right)}\right)^{\alpha_{1}+\alpha_{3}}\left(1-e^{-\lambda H\left(x_{2} ; \xi\right)}\right)^{\alpha_{2}}, \\
& b_{2}=\left(1-e^{-\lambda H\left(x_{1} ; \xi\right)}\right)^{\alpha_{1}}\left(1-e^{-\lambda H\left(x_{2} ; \xi\right)}\right)^{\alpha_{2}+\alpha_{3}}, \\
& c_{1}=F_{\mathrm{EEW}}\left(x_{1} ; \alpha_{1}+\alpha_{3}, \lambda, \xi\right) F_{\mathrm{EEW}}\left(x_{2} ; \alpha_{2}, \lambda, \xi\right), \\
& c_{2}=F_{\mathrm{EEW}}\left(x_{1} ; \alpha_{1}, \lambda, \xi\right) F_{\mathrm{EEW}}\left(x_{2} ; \alpha_{2}+\alpha_{3}, \lambda, \xi\right), \\
& c_{0}=F_{\mathrm{EEW}}\left(x ; \alpha_{1}+\alpha_{2}+\alpha_{3}, \lambda, \xi\right) .
\end{aligned}
$$

The following theorem gives the joint pdf of the random variables $X_{1}$ and $X_{2}$ which is the joint pdf of $\operatorname{BEEW}\left(\alpha_{1}, \alpha_{2}, \alpha_{3}, \lambda, \xi\right)$.
Theorem 2.2. If $\left(X_{1}, X_{2}\right) \sim \operatorname{BEEW}\left(\alpha_{1}, \alpha_{2}, \alpha_{3}, \lambda, \boldsymbol{\xi}\right)$ then the joint pdf of $\left(X_{1}, X_{2}\right)$ for $x_{1}>0, x_{2}>0$, is

$$
f_{\text {BEEW }}\left(x_{1}, x_{2}\right)=\left\{\begin{array}{lll}
f_{1}\left(x_{1}, x_{2}\right) & \text { if } & 0<x_{1}<x_{2} \\
f_{2}\left(x_{1}, x_{2}\right) & \text { if } & 0<x_{2}<x_{1} \\
f_{0}(x) & \text { if } & 0<x_{1}=x_{2}=x
\end{array}\right.
$$

where

$$
\begin{aligned}
f_{1}\left(x_{1}, x_{2}\right)= & f_{\text {EEW }}\left(x_{1} ; \alpha_{1}+\alpha_{3}, \lambda, \xi\right) f_{\text {EEW }}\left(x_{2} ; \alpha_{2}, \lambda, \xi\right) \\
= & \left(\alpha_{1}+\alpha_{3}\right) \alpha_{2} \lambda^{2} h\left(x_{1} ; \xi\right) h\left(x_{2} ; \xi\right) \\
& \times\left(1-e^{-\lambda H\left(x_{1} ; \xi\right)}\right)^{\alpha_{1}+\alpha_{3}-1} \\
& \times\left(1-e^{-\lambda H\left(x_{2} ; \xi\right)}\right)^{\alpha_{2}-1} e^{-\lambda H\left(x_{1} ; \xi\right)-\lambda H\left(x_{2} ; \xi\right)}
\end{aligned}
$$

$$
\begin{aligned}
f_{2}\left(x_{1}, x_{2}\right)= & f_{\text {EEW }}\left(x_{1} ; \alpha_{1}, \lambda, \xi\right) f_{\text {EEW }}\left(x_{2} ; \alpha_{2}+\alpha_{3}, \lambda, \xi\right) \\
= & \left(\alpha_{2}+\alpha_{3}\right) \alpha_{1} \lambda^{2} h\left(x_{1} ; \xi\right) h\left(x_{2} ; \xi\right) \\
& \times\left(1-e^{-\lambda H\left(x_{1} ; \xi\right)}\right)^{\alpha_{1}-1} \\
& \times\left(1-e^{-\lambda H\left(x_{2} ; \xi\right)}\right)^{\alpha_{2}+\alpha_{3}-1} e^{-\lambda H\left(x_{1} ; \xi\right)-\lambda H\left(x_{2} ; \xi\right)}
\end{aligned}
$$

$$
\begin{aligned}
f_{0}(x)= & \frac{\alpha_{3}}{\alpha_{1}+\alpha_{2}+\alpha_{3}} f_{\mathrm{EEW}}\left(x ; \alpha_{1}+\alpha_{2}+\alpha_{3}, \lambda, \xi\right) \\
= & \alpha_{3} \lambda h(x ; \xi)\left(1-e^{-\lambda H(x ; \xi)}\right)^{\alpha_{1}+\alpha_{2}+\alpha_{3}-1} \\
& \times e^{-\lambda H(x ; \xi)} .
\end{aligned}
$$

Proof. First assume that $x_{1}<x_{2}$. Then, the expression for $f_{1}\left(x_{1}, x_{2}\right)$ can be obtained simply by differentiating the joint cdf $F_{\mathrm{BEEW}}\left(x_{1}, x_{2}\right)$ given in (7) with respect to $x_{1}$ and $x_{2}$. Similarly, we find the expression of $f_{2}\left(x_{1}, x_{2}\right)$ when $x_{2}<x_{1}$. But $f_{0}(x)$ cannot be derived in the same way. Using the facts that

$$
\begin{aligned}
& \int_{0}^{\infty} \int_{0}^{x_{2}} f_{1}\left(x_{1}, x_{2}\right) d x_{1} d x_{2} \\
& +\int_{0}^{\infty} \int_{0}^{x_{1}} f_{2}\left(x_{1}, x_{2}\right) d x_{2} d x_{1}+\int_{0}^{\infty} f_{0}(x) d x=1, \\
& \int_{0}^{\infty} \int_{0}^{x_{2}} f_{1}\left(x_{1}, x_{2}\right) d x_{1} d x_{2}
\end{aligned}
$$$$
=\alpha_{2} \int_{0}^{\infty} \lambda h(x ; \xi)\left(1-e^{-\lambda H(x ; \xi)}\right)^{\alpha_{1}+\alpha_{2}+\alpha_{3}-1} e^{-\lambda H(x ; \xi)} d x,
$$

and

$$
\begin{aligned}
& \int_{0}^{\infty} \int_{0}^{x_{1}} f_{2}\left(x_{1}, x_{2}\right) d x_{2} d x_{1} \\
& =\alpha_{1} \int_{0}^{\infty} \lambda h(x ; \xi)\left(1-e^{-\lambda H(x ; \xi)}\right)^{\alpha_{1}+\alpha_{2}+\alpha_{3}-1} e^{-\lambda H(x ; \xi)} d x .
\end{aligned}
$$

Note that

$$
\begin{aligned}
& \int_{0}^{\infty} f_{0}(x) d x \\
& =\alpha_{3} \int_{0}^{\infty} \lambda h(x ; \xi)\left(1-e^{-\lambda H(x ; \xi)}\right)^{\alpha_{1}+\alpha_{2}+\alpha_{3}-1} e^{-\lambda H(x ; \xi)} d x
\end{aligned}
$$




$$
=\frac{\alpha_{3}}{\alpha_{1}+\alpha_{2}+\alpha_{3}} \text {. }
$$

Thus, the result follows.

The following theorem gives the marginal pdf's of $X_{1}$ and $X_{2}$.

Theorem 2.3. The marginal distributions of $X_{1}$ and $X_{2}$ are $\operatorname{EEW}\left(\alpha_{1}+\alpha_{3}, \lambda, \xi\right)$ and $\operatorname{EEW}\left(\alpha_{2}+\alpha_{3}, \lambda, \xi\right)$, respectively.

Proof. The marginal cdf for $X_{i}$ is

$$
\begin{aligned}
F_{X_{i}}\left(x_{i}\right) & =P\left(X_{i} \leq x_{i}\right) \\
& =P\left(\max \left\{U_{i}, U_{3}\right\} \leq x_{i}\right) \\
& =P\left(U_{i} \leq x_{i}, U_{3} \leq x_{i}\right) .
\end{aligned}
$$

Since the random variables $U_{i},(i=1,2)$ are mutually independent, we obtain

$$
\begin{aligned}
F_{X_{i}}\left(x_{i}\right) & =P\left(U_{i} \leq x_{i}\right) P\left(U_{3} \leq x_{i}\right) \\
& =F_{\mathrm{EEW}}\left(x_{i} ; \alpha_{i}, \lambda, \xi\right) F_{\mathrm{EEW}}\left(x_{i} ; \alpha_{3}, \lambda, \xi\right) \\
& =F_{\mathrm{EEW}}\left(x_{i} ; \alpha_{i}+\alpha_{3}, \lambda, \xi\right) .
\end{aligned}
$$

From 12, we can derive the pdf of $X_{i}$ by differentiation.

The BEEW model has both an absolute continuous part and a singular part, similar to Marshall and Olkin's bivariate exponential model. The joint cdf of $X_{1}$ and $X_{2}$ has a singular part along the line $x_{1}=x_{2}$, with weight $\frac{\alpha_{3}}{\alpha_{1}+\alpha_{2}+\alpha_{3}}$, and has an absolutely continuous part on $0<x_{1} \neq x_{2}<\infty$ with weight $\frac{\alpha_{1}+\alpha_{2}}{\alpha_{1}+\alpha_{2}+\alpha_{3}}$.

Interestingly, the BEEW model can be obtained by using the Marshall-Olkin (MO) copula with the marginals as the EEW distributions. To every bivariate cdf $F_{X_{1}, X_{2}}$ with continuous marginals $F_{X_{1}}$ and $F_{X_{2}}$ there corresponds a unique bivariate cdf with uniform margins $C:[0,1]^{2} \rightarrow$ $[0,1]$ called a copula, such that $F_{X_{1}, X_{2}}\left(x_{1}, x_{2}\right)=C\left\{F_{X_{1}}\right.$ $\left.\left(x_{1}\right), F_{X_{2}}\left(x_{2}\right)\right\}$ holds for all $\left(x_{1}, x_{2}\right) \in \mathbb{R}^{2}$ (Nelson, 1999). The MO copula is

$$
C_{\theta_{1}, \theta_{2}}\left(u_{1}, u_{2}\right)=u_{1}^{1-\theta_{1}} u_{2}^{1-\theta_{2}} \min \left\{u_{1}^{\theta_{1}}, u_{2}^{\theta_{2}}\right\}
$$

for $0<\theta_{1}<1$ and $0<\theta_{2}<1$. Using $u_{i}=F_{X_{i}}\left(x_{i}\right)$ where $X_{i}$ is $\operatorname{EEW}\left(\alpha_{i}+\alpha_{3}, \lambda, \xi\right)$ and $\theta_{i}=\frac{\alpha_{3}}{\alpha_{i}+\alpha_{3}}, i=1,2,3$, gives the same joint cdf $F_{X_{1}, X_{2}}$ as (7).

The following result will provide explicitly the absolute continuous part and the singular part of the BEEW cdf.

Theorem 2.4. If $\left(X_{1}, X_{2}\right) \sim \operatorname{BEEW}\left(\alpha_{1}, \alpha_{2}, \alpha_{3}, \lambda, \xi\right)$, then

$$
\begin{aligned}
F_{X_{1}, X_{2}}\left(x_{1}, x_{2}\right)= & \frac{\alpha_{1}+\alpha_{2}}{\alpha_{1}+\alpha_{2}+\alpha_{3}} F_{a}\left(x_{1}, x_{2}\right) \\
& +\frac{\alpha_{3}}{\alpha_{1}+\alpha_{2}+\alpha_{3}} F_{S}\left(x_{1}, x_{2}\right),
\end{aligned}
$$

where for $x=\min \left\{x_{1}, x_{2}\right\}$,

$$
F_{S}\left(x_{1}, x_{2}\right)=\left(1-e^{-\lambda H(x ; \xi)}\right)^{\alpha_{1}+\alpha_{2}+\alpha_{3}},
$$

and

$$
\begin{aligned}
F_{a}\left(x_{1}, x_{2}\right)= & \frac{\alpha_{1}+\alpha_{2}+\alpha_{3}}{\alpha_{1}+\alpha_{2}}\left(1-e^{-\lambda H\left(x_{1} ; \xi\right)}\right)^{\alpha_{1}} \\
& \times\left(1-e^{-\lambda H\left(x_{2} ; \xi\right)}\right)^{\alpha_{2}}\left(1-e^{-\lambda H(x ; \xi)}\right)^{\alpha_{3}} \\
& -\frac{\alpha_{3}}{\alpha_{1}+\alpha_{2}}\left(1-e^{-\lambda H(x ; \xi)}\right)^{\alpha_{1}+\alpha_{2}+\alpha_{3}}
\end{aligned}
$$

here $F_{S}(.,$.$) and F_{a}(.,$.$) are the singular and the absolute$ continuous parts, respectively.

Proof. To find $F_{a}\left(x_{1}, x_{2}\right)$ from $F_{X_{1}, X_{2}}\left(x_{1}, x_{2}\right)=a F_{a}\left(x_{1}, x_{2}\right)$ $+(1-a) F_{S}\left(x_{1}, x_{2}\right), 0 \leq a \leq 1$, we compute

$$
\begin{aligned}
\frac{\partial^{2} F_{X_{1}, X_{2}}\left(x_{1}, x_{2}\right)}{\partial x_{1} \partial x_{2}} & =a f_{a}\left(x_{1}, x_{2}\right) \\
& = \begin{cases}f_{1}\left(x_{1}, x_{2}\right) & \text { if } x_{1}<x_{2} \\
f_{2}\left(x_{1}, x_{2}\right) & \text { if } x_{1}>x_{2}\end{cases}
\end{aligned}
$$

from which $a$ may be obtained as

$$
\begin{aligned}
a= & \int_{0}^{\infty} \int_{0}^{x_{2}} f_{1}\left(x_{1}, x_{2}\right) d x_{1} d x_{2} \\
& +\int_{0}^{\infty} \int_{0}^{x_{1}} f_{2}\left(x_{1}, x_{2}\right) d x_{2} d x_{1}=\frac{\alpha_{1}+\alpha_{2}}{\alpha_{1}+\alpha_{2}+\alpha_{3}},
\end{aligned}
$$

and

$$
F_{a}\left(x_{1}, x_{2}\right)=\int_{0}^{x_{1}} \int_{0}^{x_{2}} f_{\mathrm{a}}(s, t) d s d t
$$

Once $a$ and $F_{a}(.,$.$) are determined, F_{s}(.,$.$) can be ob-$ tained by subtraction.

Corollary 2. The joint $p d f$ of $X_{1}$ and $X_{2}$ can be written as follows for $x=\min \left\{x_{1}, x_{2}\right\}$;

$$
\begin{aligned}
f_{X_{1}, X_{2}}\left(x_{1}, x_{2}\right)= & \frac{\alpha_{1}+\alpha_{2}}{\alpha_{1}+\alpha_{2}+\alpha_{3}} f_{a}\left(x_{1}, x_{2}\right) \\
& +\frac{\alpha_{3}}{\alpha_{1}+\alpha_{2}+\alpha_{3}} f_{s}(x),
\end{aligned}
$$

where

$$
f_{a}\left(x_{1}, x_{2}\right)=\frac{\alpha_{1}+\alpha_{2}+\alpha_{3}}{\alpha_{1}+\alpha_{2}} \times\left\{\begin{array}{lll}
d_{1} & \text { if } & x_{1}<x_{2} \\
d_{2} & \text { if } & x_{1}>x_{2}
\end{array}\right.
$$

and

$$
\begin{aligned}
& d_{1}=f_{\mathrm{EEW}}\left(x_{1} ; \alpha_{1}+\alpha_{3}, \lambda, \xi\right) f_{\mathrm{EEW}}\left(x_{2} ; \alpha_{2}, \lambda, \xi\right), \\
& d_{2}=f_{\mathrm{EEW}}\left(x_{1} ; \alpha_{1}, \lambda, \xi\right) f_{\mathrm{EEW}}\left(x_{2} ; \alpha_{2}+\alpha_{3}, \lambda, \xi\right), \\
& f_{s}(x)=f_{\mathrm{EEW}}\left(x ; \alpha_{1}+\alpha_{2}+\alpha_{3}, \lambda, \xi\right) .
\end{aligned}
$$

Clearly, here $f_{a}\left(x_{1}, x_{2}\right)$ and $f_{s}(x)$ are the absolute continuous part and singular part, respectively. 
Having obtained the marginal pdf of $X_{1}$ and $X_{2}$, we can now derive the pdf's as presented in the following theorem.

Theorem 2.5. The conditional pdf of $X_{i}$ given $X_{j}=x_{j}$, denoted by $f_{X_{i} \mid X_{j}}\left(x_{i} \mid x_{j}\right), i \neq j=1,2$, is given by

$$
f_{X_{i} \mid X_{j}}\left(x_{i} \mid x_{j}\right)=\left\{\begin{array}{lll}
f_{X_{i} \mid X_{j}}^{(1)}\left(x_{i} \mid x_{j}\right) & \text { if } & 0<x_{i}<x_{j} \\
f_{X_{i} \mid X_{j}}^{(2)}\left(x_{i} \mid x_{j}\right) & \text { if } & 0<x_{j}<x_{i} \\
f_{X_{i} \mid X_{j}}^{(3)}\left(x_{i} \mid x_{j}\right) & \text { if } & x_{i}=x_{j}>0
\end{array}\right.
$$

where

$$
\begin{aligned}
& f_{X_{i} \mid X_{j}}^{(1)}\left(x_{i} \mid x_{j}\right)=\frac{\left(\alpha_{i}+\alpha_{3}\right) \alpha_{j} \lambda h\left(x_{i} ; \xi\right)}{\left(\alpha_{2}+\alpha_{3}\right)\left(1-e^{-\lambda H\left(x_{j} ; \xi\right)}\right)^{\alpha_{3}}} \\
& \quad \times\left(1-e^{-\lambda H\left(x_{i} ; \xi\right)}\right)^{\alpha_{i}+\alpha_{3}-1} e^{-\lambda H\left(x_{i} ; \xi\right)}, \\
& f_{X_{i} \mid X_{j}}^{(2)}\left(x_{i} \mid x_{j}\right)=\alpha_{i} \lambda h\left(x_{i} ; \xi\right)\left(1-e^{-\lambda H\left(x_{i} ; \xi\right)}\right)^{\alpha_{i}-1} \\
& \quad \times e^{-\lambda H\left(x_{i} ; \xi\right)} \\
& f_{X_{i} \mid X_{j}}^{(3)}\left(x_{i} \mid x_{j}\right)=\frac{\alpha_{3}}{\alpha_{j}+\alpha_{3}}\left(1-e^{-\lambda H\left(x_{i} ; \xi\right)}\right)^{\alpha_{i}} .
\end{aligned}
$$

Proof. The proof follows readily upon substituting the joint pdf of $\left(X_{1}, X_{2}\right)$ given in Theorem 2.2 and the marginal pdf of $X_{j}$, given in Theorem 2.3, using the following relation

$$
f_{X_{i} \mid X_{j}}\left(x_{i} \mid x_{j}\right)=\frac{f_{X_{i}, X_{j}}\left(x_{i}, x_{j}\right)}{f_{X_{j}}\left(x_{j}\right)}, i=1,2 .
$$

Proposition 1. Since the joint sf and the joint cdf have the following relation

$S_{X_{1}, X_{2}}\left(x_{1}, x_{2}\right)=1-F_{X_{1}}\left(x_{1}\right)-F_{X_{2}}\left(x_{2}\right)+F_{X_{1}, X_{2}}\left(x_{1}, x_{2}\right)$,

therefore, the joint sf of $X_{1}$ and $X_{2}$ also can be expressed in a compact form.

Proposition 2. Basu (1971) defined the bivariate failure rate function $h_{X_{1}, X_{2}}\left(x_{1}, x_{2}\right)$ for the random vector $\left(X_{1}, X_{2}\right)$ as the following relation

$$
h_{X_{1}, X_{2}}\left(x_{1}, x_{2}\right)=\frac{f_{X_{1}, X_{2}}\left(x_{1}, x_{2}\right)}{S_{X_{1}, X_{2}}\left(x_{1}, x_{2}\right)} .
$$

We can obtained the bivariate failure rate function $h_{X_{1}, X_{2}}\left(x_{1}\right.$, $\left.x_{2}\right)$ for the random vector $\left(X_{1}, X_{2}\right)$ by substituting from (8) and (15) in (16).

Lemma 1. The cdf of $Y=\max \left\{X_{1}, X_{2}\right\}$ is given as

$$
F_{Y}(y)=\left(1-e^{-\lambda H(y ; \xi)}\right)^{\alpha_{1}+\alpha_{2}+\alpha_{3}} .
$$

Proof. Since

$$
\begin{aligned}
F_{Y}(y) & =P(Y \leq y)=P\left(\max \left\{X_{1}, X_{2}\right\} \leq y\right) \\
& =P\left(X_{1} \leq y, X_{2} \leq y\right) \\
& =P\left(\max \left\{U_{1}, U_{3}\right\} \leq y, \max \left\{U_{2}, U_{3}\right\} \leq y\right) \\
& =P\left(U_{1} \leq y, U_{2} \leq y, U_{3} \leq y\right),
\end{aligned}
$$

where the random variables $U_{i}(i=1,2,3)$ are mutually independent, we directly obtain the result.

Lemma 2. The cdf of $T=\min \left\{X_{1}, X_{2}\right\}$ is given as

$$
\begin{aligned}
F_{T}(t)= & \left(1-e^{-\lambda H(t ; \xi)}\right)^{\alpha_{1}+\alpha_{3}}+\left(1-e^{-\lambda H(t ; \xi)}\right)^{\alpha_{2}+\alpha_{3}} \\
& -\left(1-e^{-\lambda H(t ; \xi)}\right)^{\alpha_{1}+\alpha_{2}+\alpha_{3}} .
\end{aligned}
$$

Proof. It is easy to prove that by using Equations (15) and (17).

\section{Special cases}

In this Section, we consider some special cases of the BEEW distributions.

\subsection{Bivariate generalized exponential distri- bution}

If $H(x ; \xi)=x$, then the joint cdf (7) becomes

$$
F_{X_{1}, X_{2}}\left(x_{1}, x_{2}\right)=\left\{\begin{array}{lll}
b_{1} & \text { if } & x_{1} \leq x_{2} \\
b_{2} & \text { if } & x_{1}>x_{2}
\end{array}\right.
$$

where

$$
\begin{aligned}
& b_{1}=\left(1-e^{-\lambda x_{1}}\right)^{\alpha_{1}+\alpha_{3}}\left(1-e^{-\lambda x_{2}}\right)^{\alpha_{2}}, \\
& b_{2}=\left(1-e^{-\lambda x_{1}}\right)^{\alpha_{1}}\left(1-e^{-\lambda x_{2}}\right)^{\alpha_{2}+\alpha_{3}},
\end{aligned}
$$

which is the joint cdf of bivariate generalized exponential (BGE) distribution introduced by Kundu and Gupta (2009). By Theorem 12, the marginal distributions of $X_{1}$ and $X_{2}$ are $\operatorname{GE}\left(\alpha_{1}+\alpha_{3}, \lambda\right)$ and GE $\left(\alpha_{2}+\alpha_{3}, \lambda\right)$, respectively.

\subsection{Bivariate generalized linear failure rate distribution}

If $H(x ; \xi)=\beta x+\frac{\gamma}{2} x^{2}$ and $\lambda=1$, then the joint cdf (7) becomes

$$
F_{X_{1}, X_{2}}\left(x_{1}, x_{2}\right)=\left\{\begin{array}{lll}
b_{1} & \text { if } & x_{1} \leq x_{2} \\
b_{2} & \text { if } & x_{1}>x_{2}
\end{array}\right.
$$

where

$$
b_{1}=\left(1-e^{-\beta x_{1}-\frac{\gamma}{2} x_{1}^{2}}\right)^{\alpha_{1}+\alpha_{3}}\left(1-e^{-\beta x_{2}-\frac{\gamma}{2} x_{2}^{2}}\right)^{\alpha_{2}},
$$




$$
b_{2}=\left(1-e^{-\beta x_{1}-\frac{\gamma}{2} x_{1}^{2}}\right)^{\alpha_{1}}\left(1-e^{-\beta x_{2}-\frac{\gamma}{2} x_{2}^{2}}\right)^{\alpha_{2}+\alpha_{3}},
$$

which is the joint cdf of bivariate generalized linear failure rate (BGLFR) distribution introduced by Sarhan et al. (2011). By Theorem 12, the marginal distributions of $X_{1}$ and $X_{2}$ are $\operatorname{GLFR}\left(\alpha_{1}+\alpha_{3}, \beta, \gamma\right)$ and $\operatorname{GLFR}\left(\alpha_{2}+\alpha_{3}\right.$, $\beta, \gamma)$, respectively.

\subsection{Bivariate exponentiated Weibull distri- bution}

If $H(x ; \xi)=x^{\beta}$, then the joint cdf (7) becomes

$$
F_{X_{1}, X_{2}}\left(x_{1}, x_{2}\right)=\left\{\begin{array}{lll}
b_{1} & \text { if } x_{1} \leq x_{2} \\
b_{2} & \text { if } x_{1}>x_{2}
\end{array}\right.
$$

where

$$
\begin{aligned}
& b_{1}=\left(1-e^{-\lambda x_{1}^{\beta}}\right)^{\alpha_{1}+\alpha_{3}}\left(1-e^{-\lambda x_{2}^{\beta}}\right)^{\alpha_{2}}, \\
& b_{2}=\left(1-e^{-\lambda x_{1}^{\beta}}\right)^{\alpha_{1}}\left(1-e^{-\lambda x_{2}^{\beta}}\right)^{\alpha_{2}+\alpha_{3}} .
\end{aligned}
$$

We call this, bivariate exponentiated Weibull (BEW) distribution. By Theorem 12, the marginal distributions of $X_{1}$ and $X_{2}$ are $\operatorname{EW}\left(\alpha_{1}+\alpha_{3}, \lambda, \beta\right)$ and $\operatorname{EW}\left(\alpha_{2}+\alpha_{3}, \lambda, \beta\right)$, respectively.

\subsection{Bivariate generalized Gompertz distri- bution}

If $H(x ; \xi)=\beta^{-1}\left(e^{\beta x}-1\right)$, then the joint cdf (7) becomes

$$
F_{X_{1}, X_{2}}\left(x_{1}, x_{2}\right)=\left\{\begin{array}{lll}
b_{1} & \text { if } & x_{1} \leq x_{2} \\
b_{2} & \text { if } & x_{1}>x_{2}
\end{array}\right.
$$

where

$$
\begin{aligned}
& b_{1}=\left(1-e^{-\lambda \beta^{-1}\left(e^{\beta x_{1}}-1\right)}\right)^{\alpha_{1}+\alpha_{3}}\left(1-e^{-\lambda \beta^{-1}\left(e^{\beta x_{2}}-1\right)}\right)^{\alpha_{2}}, \\
& b_{2}=\left(1-e^{-\lambda \beta^{-1}\left(e^{\beta x_{1}}-1\right)}\right)^{\alpha_{1}}\left(1-e^{-\lambda \beta^{-1}\left(e^{\beta x_{2}}-1\right)}\right)^{\alpha_{2}+\alpha_{3}},
\end{aligned}
$$

which is the joint cdf of bivariate generalized Gompertz (BGG) distribution introduced by El-Sherpieny et al. (2013). By Theorem 12, the marginal distributions of $X_{1}$ and $X_{2}$ are GG $\left(\alpha_{1}+\alpha_{3}, \lambda, \beta\right)$ and GG $\left(\alpha_{2}+\alpha_{3}, \lambda, \beta\right)$, respectively.

\subsection{Bivariate exponentiated generalized -Weibull - Gompertz distribution}

If $H(x ; \xi)=x^{\beta}\left(e^{\gamma x^{\delta}}-1\right)$, then the joint cdf (7) becomes

$$
F_{X_{1}, X_{2}}\left(x_{1}, x_{2}\right)=\left\{\begin{array}{lll}
b_{1} & \text { if } & x_{1} \leq x_{2} \\
b_{2} & \text { if } & x_{1}>x_{2}
\end{array}\right.
$$

where

$$
\begin{aligned}
& b_{1}=\left(1-e^{-\lambda x_{1}^{\beta}\left(e^{\gamma x_{1}^{\delta}}-1\right)}\right)^{\alpha_{1}+\alpha_{3}}\left(1-e^{-\lambda x_{2}^{\beta}\left(e^{\gamma x_{2}^{\delta}}-1\right)}\right)^{\alpha_{2}}, \\
& b_{2}=\left(1-e^{-\lambda x_{1}^{\beta}\left(e^{\gamma x_{1}^{\delta}}-1\right)}\right)^{\alpha_{1}}\left(1-e^{-\lambda x_{2}^{\beta}\left(e^{\gamma x_{2}^{\delta}}-1\right)}\right)^{\alpha_{2}+\alpha_{3}},
\end{aligned}
$$

which is the joint cdf of bivariate exponentiated generalized Weibull-Gompertz (BEGWG) distribution introduced by El-Bassioun et al. (2015). By Theorem 12, the marginal distributions of $X_{1}$ and $X_{2}$ are EGWG $\left(\alpha_{1}+\right.$ $\left.\alpha_{3}, \lambda, \beta, \gamma, \delta\right)$ and EGWG $\left(\alpha_{2}+\alpha_{3}, \lambda, \beta, \gamma, \delta\right)$, respectively.

\subsection{Bivariate exponentiated modified Weibull extension distribution}

If $H(x ; \xi)=\beta\left(e^{(x / \beta)^{\gamma}}-1\right)$, then the joint cdf (7) becomes

$$
F_{X_{1}, X_{2}}\left(x_{1}, x_{2}\right)=\left\{\begin{array}{lll}
b_{1} & \text { if } & x_{1} \leq x_{2} \\
b_{2} & \text { if } & x_{1}>x_{2}
\end{array}\right.
$$

where

$$
\begin{aligned}
& b_{1}=\left(1-e^{-\lambda \beta\left(e^{\left(x_{1} / \beta\right)^{\gamma}}-1\right)}\right)^{\alpha_{1}+\alpha_{3}}\left(1-e^{-\lambda \beta\left(e^{\left(x_{2} / \beta\right)^{\gamma}}-1\right)}\right)^{\alpha_{2}}, \\
& b_{2}=\left(1-e^{-\lambda \beta\left(e^{\left(x_{1} / \beta\right)^{\gamma}}-1\right)}\right)^{\alpha_{1}}\left(1-e^{-\lambda \beta\left(e^{\left(x_{2} / \beta\right)^{\gamma}}-1\right)}\right)^{\alpha_{2}+\alpha_{3}},
\end{aligned}
$$

which is the joint cdf of bivariate exponentiated modified Weibull extension (BEMWE) distribution introduced by El-Gohary and El-Morshedy (2015). By Theorem 12, the marginal distributions of $X_{1}$ and $X_{2}$ are $\operatorname{EMWE}\left(\alpha_{1}+\right.$ $\left.\alpha_{3}, \lambda, \beta, \gamma\right)$ and EMWE $\left(\alpha_{2}+\alpha_{3}, \lambda, \beta, \gamma\right)$, respectively.

\section{Maximum likelihood estimation}

In this section, we first study the maximum likelihood estimations (MLE's) of the parameters. Then, we propose an Expectation-Maximization (EM) algorithm to estimate the parameters.

Let $\left(x_{11}, x_{12}\right), \ldots,\left(x_{1 n}, x_{2 n}\right)$ be an observed sample with size $n$ from BEEW distribution with parameters $\boldsymbol{\Theta}=\left(\alpha_{1}, \alpha_{2}, \alpha_{3}, \lambda, \zeta\right)^{\prime}$. Also, consider

$$
\begin{aligned}
& I_{0}=\left\{i: x_{1 i}=x_{2 i}=x_{i}\right\}, \quad I_{1}=\left\{i: x_{1 i}<x_{2 i}\right\}, \\
& I_{2}=\left\{i: x_{1 i}>x_{2 i}\right\}, \quad i=1, \ldots, n \text {, }
\end{aligned}
$$

and

$n_{0}=\left|I_{0}\right|, \quad n_{1}=\left|I_{1}\right|, \quad n_{2}=\left|I_{2}\right|, \quad n=n_{0}+n_{1}+n_{2}$.

Therefore, the log-likelihood function can be written as

$$
\begin{aligned}
\ell(\boldsymbol{\Theta})= & \sum_{i \in I_{1}} \log \left(f_{1}\left(x_{1 i}, x_{2 i}\right)\right)+\sum_{i \in I_{2}} \log \left(f_{2}\left(x_{1 i}, x_{2 i}\right)\right) \\
& +\sum_{i \in I_{0}} \log \left(f_{0}\left(x_{i}\right)\right)
\end{aligned}
$$




$$
\begin{aligned}
= & \left(2 n_{1}+2 n_{2}+n_{0}\right) \log (\lambda)+n_{1} \log \left(\alpha_{2}\right) \\
& +n_{2} \log \left(\alpha_{1}\right)+n_{0} \log \left(\alpha_{3}\right) \\
& +n_{1} \log \left(\alpha_{1}+\alpha_{3}\right)+n_{2} \log \left(\alpha_{2}+\alpha_{3}\right) \\
& +\sum_{i \in I_{1} \cup I_{2}} \log \left(h\left(x_{1 i} ; \xi\right)\right) \\
& +\sum_{i \in I_{1} \cup I_{2}} \log \left(h\left(x_{2 i} ; \xi\right)\right)+\sum_{i \in I_{0}} \log \left(h\left(x_{i} ; \xi\right)\right) \\
& +\left(\alpha_{1}+\alpha_{3}-1\right)\left(\sum_{i \in I_{1}} \log \left(1-e^{-\lambda H\left(x_{1 i} ; \xi\right)}\right)\right. \\
& \left.+\sum_{i \in I_{2}} \log \left(1-e^{-\lambda H\left(x_{2 i} ; \xi\right)}\right)\right) \\
& +\left(\alpha_{2}-1\right) \sum_{i \in I_{1}} \log \left(1-e^{-\lambda H\left(x_{2 i} ; \xi\right)}\right) \\
& +\left(\alpha_{1}-1\right) \sum_{i \in I_{2}} \log \left(1-e^{-\lambda H\left(x_{1 i} ; \xi\right)}\right) \\
& +\left(\alpha_{1}+\alpha_{2}+\alpha_{3}-1\right) \sum_{i \in I_{0}} \log \left(1-e^{-\lambda H\left(x_{i} ; \xi\right)}\right) \\
& +\lambda\left(\sum_{i \in I_{0}} x_{i}+\sum_{i \in I_{1} \cup I_{2}} x_{1 i}+\sum_{i \in I_{1} \cup I_{2}} x_{2 i}\right)
\end{aligned}
$$

where $f_{1}, f_{2}$ and $f_{0}$ are given in (9), (10) and (11), respectively. We can obtain the MLE's of the parameters by maximizing $\ell(\boldsymbol{\Theta})$ in (18) with respect to the unknown parameters. This is clearly a $(k+4)$-dimensional problem. However, no explicit expressions are available for the MLE's. We need to solve $(k+4)$ non-linear equations simultaneously, which may not be very simple. Therefore, we present an expectation-maximization (EM) algorithm to find the MLE's of parameters. It may be noted that if instead of $\left(X_{1}, X_{2}\right)$, we observe $U_{1}, U_{2}$, and $U_{3}$, the MLE's of the parameters can be obtained by solving a two-dimensional optimization process, which is clearly much more convenient than solving a $(k+4)$ dimensional optimization process. For this reason, we treat this problem as a missing value problem.

Assumed that for the bivariate random vector $\left(X_{1}\right.$, $X_{2}$ ), there is an associated random vectors

$\Lambda_{1}=\left\{\begin{array}{ll}0 & U_{1}>U_{3} \\ 1 & U_{1}<U_{3}\end{array} \quad\right.$ and $\quad \Lambda_{2}=\left\{\begin{array}{cc}0 & U_{2}>U_{3} \\ 1 & U_{2}<U_{3} .\end{array}\right.$

Note that if $X_{1}=X_{2}$, then $\Lambda_{1}=\Lambda_{2}=0$. But if $X_{1}<$ $X_{2}$ or $X_{1}>X_{2}$, then $\left(\Lambda_{1}, \Lambda_{2}\right)$ is missing. If $\left(X_{1}, X_{2}\right) \in I_{1}$ then the possible values of $\left(\Lambda_{1}, \Lambda_{2}\right)$ are $(1,0)$ or $(1,1)$, and If $\left(X_{1}, X_{2}\right) \in I_{2}$ then the possible values of $\left(\Lambda_{1}, \Lambda_{2}\right)$ are $(0,1)$ or $(1,1)$ with non-zero probabilities.

Now, we are in a position to provide the EM algorithm. In the E-step of the EM-algorithm, we treat it as complete observation when they belong to $I_{0}$. If the observation belong to $I_{1}$, we form the 'pseudo' loglikelihood function by fractioning $\left(x_{1}, x_{2}\right)$ to two partially complete "pseudo" observations of the form $\left(x_{1}, x_{2}\right.$,
$\left.u_{1}(\boldsymbol{\Theta})\right)$ and $\left(x_{1}, x_{2}, u_{2}(\boldsymbol{\Theta})\right)$, where $u_{1}(\boldsymbol{\Theta})$ and $u_{2}(\boldsymbol{\Theta})$ are the conditional probabilities that $\left(\Lambda_{1}, \Lambda_{2}\right)$ takes values $(1,0)$ and $(1,1)$, respectively. It is clear that

$$
u_{1}(\boldsymbol{\Theta})=\frac{\alpha_{1}}{\alpha_{1}+\alpha_{3}}, \quad u_{2}(\boldsymbol{\Theta})=\frac{\alpha_{3}}{\alpha_{1}+\alpha_{3}} .
$$

Similarly, If the observation belong to $I_{2}$, we form the 'pseudo' log-likelihood function of the from $\left(y_{1}, y_{2}\right.$, $\left.v_{1}(\boldsymbol{\Theta})\right)$ and $\left(x_{1}, x_{2}, v_{2}(\boldsymbol{\Theta})\right)$, where $v_{1}(\boldsymbol{\Theta})$ and $v_{2}(\boldsymbol{\Theta})$ are the conditional probabilities that $\left(\Lambda_{1}, \Lambda_{2}\right)$ takes values $(0,1)$ and $(1,1)$, respectively. Therefore,

$$
v_{1}(\boldsymbol{\Theta})=\frac{\alpha_{2}}{\alpha_{2}+\alpha_{3}}, \quad v_{2}(\boldsymbol{\Theta})=\frac{\alpha_{3}}{\alpha_{2}+\alpha_{3}} .
$$

For brevity, we write $u_{1}(\boldsymbol{\Theta}), u_{2}(\boldsymbol{\Theta}), v_{1}(\boldsymbol{\Theta}), v_{2}(\boldsymbol{\Theta})$ as $u_{1}, u_{2}, v_{1}, v_{2}$, respectively.

E-step: Consider $b_{i}=E\left(N \mid y_{1 i}, y_{2 i}, \mathbf{\Theta}\right)$. The log- likelihood function without the additive constant can be written as follows:

$$
\begin{aligned}
\ell_{\text {pseudo }}(\boldsymbol{\Theta})= & \left.n_{0}+2 n_{1}+2 n_{2}\right) \log (\lambda) \\
& +\left(u_{1} n_{1}+n_{2}\right) \log \left(\alpha_{1}\right) \\
& +\left(n_{1}+v_{1} n_{2}\right) \log \left(\alpha_{2}\right) \\
& +\left(n_{0}+u_{2} n_{1}+v_{2} n_{2}\right) \log \left(\alpha_{3}\right) \\
& +\sum_{i \in I_{0}} \log \left(h\left(x_{i} ; \xi\right)\right)+\sum_{i \in I_{1} \cup I_{2}} \log \left(h\left(x_{1 i} ; \xi\right)\right) \\
& +\sum_{i \in I_{1} \cup I_{2}} \log \left(h\left(x_{2 i} ; \xi\right)\right) \\
& +\left(\alpha_{1}+\alpha_{2}+\alpha_{3}-1\right) \sum_{i \in I_{0}} \log \left(1-e^{-\lambda H\left(x_{i} ; \xi\right)}\right) \\
& +\left(\alpha_{1}+\alpha_{3}-1\right) \sum_{i \in I_{1}} \log \left(1-e^{-\lambda H\left(x_{1 i} ; \xi\right)}\right) \\
& +\left(\alpha_{2}+\alpha_{3}-1\right) \sum_{i \in I_{2}} \log \left(1-e^{-\lambda H\left(x_{2 i} ; \xi\right)}\right) \\
& +\left(\alpha_{2}-1\right) \sum_{i \in I_{1}} \log \left(1-e^{-\lambda H\left(x_{2 i} ; \xi\right)}\right) \\
& +\left(\alpha_{1}-1\right) \sum_{i \in I_{2}} \log \left(1-e^{-\lambda H\left(x_{1 i} ; \xi\right)}\right) \\
& -\lambda\left(\sum_{i \in I_{0}} H\left(x_{i} ; \xi\right)+\sum_{i \in I_{1} \cup I_{2}} H\left(x_{1 i} ; \xi\right)\right. \\
& \left.+\sum_{i \in I_{1} \cup I_{2}} H\left(x_{2 i} ; \xi\right)\right)
\end{aligned}
$$

M-step: At this step, $\ell_{\text {pseudo }}(\boldsymbol{\Theta})$ is maximized with respect to $\alpha_{1}, \alpha_{2}, \alpha_{3}, \lambda$ and $\xi$. For fixed $\lambda$ and $\xi$, the maximization occurs at

$$
\begin{aligned}
\hat{\alpha}_{1}(\lambda, \boldsymbol{\xi}) & =\frac{u_{1} n_{1}+n_{2}}{\sum_{i \in I_{0}} W\left(x_{i}\right)+\sum_{i \in I_{1} \cup I_{2}} W\left(x_{1 i}\right)}, \\
\hat{\alpha}_{2}(\lambda) & =\frac{n_{1}+v_{1} n_{2}}{\sum_{i \in I_{0}} W\left(x_{i}\right)+\sum_{i \in I_{1} \cup I_{2}} W\left(x_{2 i}\right)},
\end{aligned}
$$




$$
\hat{\alpha}_{3}(\lambda)=\frac{n_{0}+u_{2} n_{1}+v_{2} n_{2}}{\sum_{i \in I_{0}} W\left(x_{i}\right)+\sum_{i \in I_{1}} W\left(x_{1 i}\right)+\sum_{i \in I_{2}} W\left(x_{2 i}\right)},
$$

where $W(x)=\log \left(1-e^{-\lambda H(x ; \xi)}\right)$. For fixed $\alpha_{1}, \alpha_{2}, \alpha_{3}$ and $\xi, \ell_{\text {pseudo }}(\boldsymbol{\Theta})$ is maximized with respect to $\lambda$ as a solution of the following equation:

$$
\frac{n_{0}+2 n_{1}+2 n_{2}}{\mathrm{~g}(\lambda)}=\lambda
$$

where

$$
\begin{aligned}
\mathrm{g}(\lambda)= & -\left(\alpha_{1}+\alpha_{2}+\alpha_{3}-1\right) \sum_{i \in I_{0}} \frac{H\left(x_{i} ; \xi\right) e^{-\lambda H\left(x_{i} ; \xi\right)}}{1-e^{-\lambda H\left(x_{i} ; \xi\right)}} \\
& -\left(\alpha_{1}+\alpha_{3}-1\right) \sum_{i \in I_{1}} \frac{H\left(x_{1 i} ; \xi\right) e^{-\lambda H\left(x_{1 i} ; \xi\right)}}{1-e^{-\lambda H\left(x_{1 i} ; \xi\right)}} \\
& -\left(\alpha_{2}+\alpha_{3}-1\right) \sum_{i \in I_{2}} \frac{H\left(x_{2 i} ; \xi\right) e^{-\lambda H\left(x_{2 i} ; \xi\right)}}{1-e^{-\lambda H\left(x_{2 i} ; \xi\right)}} \\
& -\left(\alpha_{2}-1\right) \sum_{i \in I_{1}} \frac{H\left(x_{2 i} ; \xi\right) e^{-\lambda H\left(x_{2 i} ; \xi\right)}}{1-e^{-\lambda H\left(x_{2 i} ; \xi\right)}} \\
& -\left(\alpha_{1}-1\right) \sum_{i \in I_{2}} \frac{H\left(x_{1 i} ; \xi\right) e^{-\lambda H\left(x_{1 i} ; \xi\right)}}{1-e^{-\lambda H\left(x_{1 i} ; \xi\right)}} \\
& +\sum_{i \in I_{0}} H\left(x_{i} ; \xi\right)+\sum_{i \in I_{1} \cup I_{2}} H\left(x_{1 i} ; \xi\right) \\
& +\sum_{i \in I_{1} \cup I_{2}} H\left(x_{2 i} ; \xi\right) .
\end{aligned}
$$

Finally, for fixed $\alpha_{1}, \alpha_{2}, \alpha_{3}$ and $\lambda, \ell_{\text {pseudo }}(\boldsymbol{\Theta})$ is maximized with respect to $\xi$ as a solution of the following equation:

$$
\frac{\partial}{\partial \xi} \ell_{\text {pseudo }}(\boldsymbol{\Theta})=\mathbf{0}
$$

The following steps can be used to compute the MLE's of the parameters via the EM algorithm:

Step 1: Take some initial value of $\boldsymbol{\Theta}$, say $\boldsymbol{\Theta}^{(0)}=\left(\alpha_{1}^{(0)}\right.$, $\left.\alpha_{2}^{(0)}, \alpha_{3}^{(0)}, \lambda^{(0)}, \boldsymbol{\xi}^{(0)}\right)^{\prime}$.

Step 2: Compute $u_{1}, u_{2}, v_{1}$, and $v_{2}$.

Step 3: Find $\hat{\lambda}$ by solving the equation (22), say $\hat{\lambda}^{(1)}$.

Step 4: Find $\hat{\xi}$ by solving the equation (23), say $\hat{\mathcal{\xi}}^{(1)}$.

Step 5: Compute $\hat{\alpha}_{i}^{(1)}=\hat{\alpha}_{i}\left(\hat{\lambda}^{(1)}, \hat{\xi}^{(1)}\right), i=1,2,3$ from (19)-(21).

Step 6: Replace $\Theta^{(0)}$ by $\hat{\Theta}^{(1)}=\left(\hat{\alpha}_{1}^{(1)}, \hat{\alpha}_{2}^{(1)}, \hat{\alpha}_{3}^{(1)}, \hat{\lambda}^{(1)}, \hat{\mathcal{\xi}}^{(1)}\right)$, go back to step 1 and continue the process until convergence take place.

\section{Two real examples}

We consider BEEW distributions for fitting these two data sets. But, this family of distributions is a large class of distributions. Here, we consider six sub-models of
BEEW distributions: BGE, BGLFR, BEW, BGG, BEGWG, and BEMWE. Some of them are suggested in literature.

Using the proposed EM algorithm, these models are fitted to the bivariate data set, and the MLE's and their corresponding log-likelihood values are calculated. The standard errors (s.e.) based on the observed information matrix are obtained.

For each fitted model, the Akaike Information Criterion (AIC), the corrected Akaike information criterion (AICC) and the Bayesian information criterion (BIC) are calculated. We also obtain the Kolmogorov-Smirnov (KS) distances between the fitted distribution, the empirical distribution function, and the corresponding $\mathrm{p}$-values (in parenthesis) for $X_{1}, X_{2}$ and $\max \left(X_{1}, X_{2}\right)$. For more information about this criteria of model selection, we refer the reader to Burnham and Anderson (1998) and Sullivan and Joyce (2005) Finally, we make use the likelihood ratio test (LRT) and the corresponding p-values for testing the BGE against other models.

Example 1. The data set is given from Meintanis (2007) and is obtained from the group stage of the UEFA Champion's League for the years 2004-05 and 2005-2006. In addition, Kundu and Gupta (2009) and Sarhan et al. (2011) analyzed this data. The data represent the football (soccer) data where at least one goal scored by the home team and at least one goal scored directly from a kick goal (like penalty kick, foul kick or any other direct kick) by any team have been considered. Here $X_{1}$ represents the time in minutes of the first kick goal scored by any team and $X_{2}$ represents the first goal of any type scored by the home team.

In Table 2 we provide the MLEs of the unknown parameters of six sub models of BEEW distributions. We have also included the AIC, AICC, and BIC values for model selection purposes. From this data, we find the values of the all unknown parameters with its standard errors and the log-likelihood for the six models. Using the EM algorithm we obtain the MLEs of all parameters. By substituting the MLE of unknown parameters, we get the estimation of the variance covariance matrix. The likelihood ratio test statistic and the associated $p$ values for marginal distributions showed that all marginal distributions provide significant fit to this data. It is clear that all six models are appropriate for this data set but among these all six models, the BEW and BGG models are preferable, on the basis of both AIC, AICC, and BIC values. The AIC, AICC, and BIC values for BEW (BGG) models are 593.361 (593.710), 595.297 (595.646), and 601.416 (601.765), respectively.

Example 2. The data set was first published in 'Washington Post' and is available in Csörgö and Welsh (1989). It is represent the American Football League for the matches on three consecutive weekends in 1986 . Here, $X_{1}$ represents the 'game time' to the first points scored by kicking the ball between 
Table 2: The MLE's, log-likelihood, AIC, AICC, BIC, K-S, and LRT statistics for six sub-models of BEEW distribution of first data set.

\begin{tabular}{|c|c|c|c|c|c|c|}
\hline & \multicolumn{6}{|c|}{ Distribution } \\
\hline Statistic & BGE & BGLFR & $\overline{B E W}$ & $\overline{\text { BGG }}$ & BEWG & BEMWE \\
\hline$\hat{\alpha}_{1}$ & 1.4452 & 0.4920 & 0.2179 & 0.6596 & 0.2474 & 0.1574 \\
\hline (s.e.) & $(0.4160)$ & $(0.0810)$ & $(0.6663)$ & $(0.2559)$ & $(0.1185)$ & $(0.2276)$ \\
\hline$\hat{\alpha}_{2}$ & 0.4681 & 0.1661 & 0.0770 & 0.2366 & 0.0896 & 0.0573 \\
\hline (s.e.) & $(0.1879)$ & $(0.0535)$ & $(0.2219)$ & $(0.1093)$ & $(0.0498)$ & $(0.0833)$ \\
\hline$\hat{\alpha}_{3}$ & 1.1704 & 0.4110 & 0.1880 & 0.5821 & 0.2223 & 0.1419 \\
\hline (s.e.) & $(0.2866)$ & $(0.0331)$ & $(0.3446)$ & $(0.1964)$ & $(0.1016)$ & $(0.2009)$ \\
\hline$\hat{\lambda}$ & 0.0390 & - & $1.914 \mathrm{e}-4$ & 0.0098 & 0.1622 & 0.0246 \\
\hline (s.e.) & $(0.0056)$ & - & $(1.83 e-5)$ & $(0.0061)$ & $(0.6398)$ & $(0.0526)$ \\
\hline$\hat{\beta}$ & - & $1.990 \mathrm{e}-4$ & 3.7136 & 0.0304 & 0.4168 & 85.9181 \\
\hline (s.e.) & 一 & $1.237 \mathrm{e}-4$ & $(0.2811)$ & $(0.0112)$ & $(0.9648)$ & (34.1193) \\
\hline$\hat{\gamma}$ & - & $7.971 \mathrm{e}-4$ & - & - & $2.624 \mathrm{e}-5$ & 4.5054 \\
\hline (s.e.) & - & $1.497 \mathrm{e}-4$ & - & - & $7.304 e-5$ & $(2.0339)$ \\
\hline$\hat{\delta}$ & - & - & - & - & 2.4645 & - \\
\hline (s.e.) & - & - & - & - & $(0.5969)$ & - \\
\hline$-\log (\ell)$ & 296.901 & 293.376 & 291.681 & 291.855 & 291.132 & 290.981 \\
\hline AIC & 601.801 & 596.752 & 593.361 & 593.710 & 596.263 & 593.962 \\
\hline $\mathrm{AICC}$ & 603.051 & 598.688 & 595.297 & 595.646 & 600.125 & 596.762 \\
\hline $\mathrm{BIC}$ & 608.245 & 604.807 & 601.416 & 601.765 & 607.540 & 603.628 \\
\hline $\mathrm{K}-\mathrm{S}\left(\mathrm{X}_{1}\right)$ & 0.1034 & 0.07082 & 0.0962 & 0.1042 & 0.1140 & 0.1182 \\
\hline (p-value) & $(0.8240)$ & $(0.9925)$ & $(0.8829)$ & $(0.8157)$ & $(0.7218)$ & $(0.6789)$ \\
\hline $\mathrm{K}-\mathrm{S}\left(X_{2}\right)$ & 0.1001 & 0.0968 & 0.1167 & 0.1243 & 0.1196 & 0.1187 \\
\hline (p-value) & $(0.8527)$ & $(0.8786)$ & $(0.6939)$ & $(0.6161)$ & $(0.6644)$ & $(0.6738)$ \\
\hline $\mathrm{K}-\mathrm{S}\left(\max \left(X_{1}, X_{2}\right)\right)$ & 0.1431 & 0.1104 & 0.0942 & 0.0984 & 0.1272 & 0.1366 \\
\hline (p-value) & $(0.4344)$ & $(0.7574)$ & 0.8978 & $(0.8661)$ & $(0.5865)$ & $(0.4940)$ \\
\hline LRT & - & 7.050 & 10.440 & 10.092 & 11.538 & 11.840 \\
\hline (p-value) & - & $(0.0079)$ & $(0.0012)$ & $(0.0015)$ & $(0.0091)$ & $(0.0026)$ \\
\hline
\end{tabular}

goal posts, and represents the 'game time' to the first points scored by moving the ball into the end zone. Kundu and Gupta (2010) Jamalizadeh and Kundu (2013), and Balakrishna and Shiji (2014) analyzed this data. We divided all the data by 100.

Table 3 displays the MLE's of the parameters (with corresponding standard errors in parentheses) for the bivariate distributions which are obtained by the EM algorithm given in Section 4. To test the goodness-of fit of the marginal distributions, we calculated the Kolmogorov - Smirnov (K-S) statistic with its respective p-value. From K-S in this table, it can be concluded that all marginal distributions of six models are appropriate for this data set. Since the values of the AIC (-65.340), BIC (-64.258), and AICC (-58.389) are smaller for the BGE distribution compared with those values of the other models, this bivariate distribution seems to be a very competitive model for these data. 
Table 3: The MLE's, log-likelihood, AIC, AICC, BIC, K-S, and LRT statistics for six sub-models of BEEW distribution of second data set.

\begin{tabular}{c|c|c|c|c|c|c|}
\hline & \multicolumn{7}{|c|}{ Distribution } \\
\hline Statistic & BGE & BGLFR & BEW & BGG & BEWG & BEMWE \\
\hline$\hat{\alpha}_{1}$ & 0.0921 & 0.0921 & 0.1367 & 0.0921 & 0.1501 & 0.1374 \\
$($ s.e. $)$ & $(0.0653)$ & $(0.0667)$ & $(0.1351)$ & $(0.0653)$ & $(0.2570)$ & $(0.1355)$ \\
\hline$\hat{\alpha}_{2}$ & 0.5722 & 0.5722 & 0.8483 & 0.5722 & 0.9313 & 0.8523 \\
$($ s.e. $)$ & $(0.1614)$ & $(0.1824)$ & $(0.6283)$ & $(0.1614)$ & $(1.4720)$ & $(0.6290)$ \\
\hline$\hat{\alpha}_{3}$ & 1.1519 & 1.1519 & 1.7113 & 1.1519 & 1.8788 & 1.7195 \\
$($ s.e. $)$ & $(0.2388)$ & $(0.2945)$ & $(1.2318)$ & $(0.2388)$ & $(2.9542)$ & $(1.2328)$ \\
\hline$\hat{\lambda}$ & 9.6187 & - & 8.5587 & 9.6187 & 3.4632 & 3.0614 \\
$($ s.e. $)$ & $(1.5569)$ & - & $(1.9069)$ & $(1.5590)$ & $(2.8867)$ & $(9.3275)$ \\
\hline$\hat{\beta}$ & - & 9.6187 & 0.8117 & $2.1 \mathrm{e}-12$ & 0.5548 & 211.651 \\
$($ s.e. $)$ & - & $(2.7455)$ & $(0.2828)$ & $(0.0455)$ & $(0.0328)$ & $(88.5725)$ \\
\hline$\hat{\gamma}$ & - & $2.351 \mathrm{e}-4$ & - & - & 1.2553 & 0.8088 \\
$($ s.e. $)$ & - & $1.297 \mathrm{e}-4$ & - & - & $(0.8749)$ & $(0.2814)$ \\
\hline$\hat{\delta}$ & - & - & - & - & 0.1462 & - \\
$($ s.e. $)$ & - & - & - & - & $(1.9357)$ & - \\
\hline $\log (\ell)$ & 36.670 & 36.670 & 36.857 & 36.670 & 36.859 & 36.857 \\
AIC & -65.340 & -63.340 & -63.714 & -63.340 & -59.717 & -61.714 \\
AICC & -64.258 & -61.673 & -62.048 & -61.673 & -56.423 & -59.314 \\
BIC & -58.389 & -54.652 & -55.026 & -54.651 & -47.553 & -51.288 \\
\hline K-S $\left(X_{1}\right)$ & 0.1808 & 0.1808 & 0.1678 & 0.1808 & 0.1679 & 0.1680 \\
$($ p-value $)$ & $(0.1282)$ & $(0.1282)$ & $(0.1872)$ & $(0.1282)$ & $(0.1869)$ & $(0.1866)$ \\
\hline K-S $\left(X_{2}\right)$ & 0.1410 & 0.1411 & 0.1289 & 0.1410 & 0.1290 & 0.1291 \\
$($ p-value) & $(0.3408)$ & $(0.3408)$ & $(0.4499)$ & $(0.3408)$ & $(0.4490)$ & $(0.4484)$ \\
\hline K-S (max $\left.\left(X_{1}, X_{2}\right)\right)$ & 0.1350 & 0.1350 & 0.1197 & 0.1350 & 0.1198 & 0.1198 \\
$($ p-value $)$ & $(0.3929)$ & $(0.3929)$ & $(0.5438)$ & $(0.3929)$ & $(0.5428)$ & $(0.5422)$ \\
\hline LRT & - & 0.000 & 0.374 & 0.000 & 0.378 & 0.374 \\
$($ p-value) & - & 1.0000 & $(0.5408)$ & 1.0000 & 0.9447 & 0.8294 \\
\hline
\end{tabular}

\section{Conclusions}

In this paper we have introduced the new bivariate exponentiated extended Weibull distribution whose marginal are exponentiated extended Weibull distributions. It contains a number of known special submodels (at least 46 bivariate distributions) such as bivariate generalized exponential, bivariate generalized linear failure rate, bivariate exponentiated Weibull, bivariate generalized Gompertz, and bivariate exponentiated modified Weibull extension distributions, among others. We think the formulas derived are manageable by using modern computer resources with analytic and numerical capabilities. The proposed bivariate model has a singular distribution, and it can be used quite effectively instead of the Marshall-Olkin bivariate exponential model or the bivariate generalized exponential model when there are ties in the data. This new bivariate distribution has several interesting properties and it can be used as an alternative to the several bivariate distributions. The generation of random samples from proposed bivariate distribution is very simple, and therefore Monte Carlo simulation can be performed very easily for different statistical inference purpose. Maximum likelihood estimates of the new bivariate model are discussed. It may be mentioned that an EM algorithm along the same lines as the bivariate case may be developed. Alternatively, using the copula structure, other estimators may be used and their properties can be established. Analyses of two real data sets indicate the good performance and usefulness of the new model. 


\section{Acknowledgments}

The authors wish to thank the Editor and two anonymous referees for their careful reading and some constructive criticism on the earlier draft of the paper. The authors are also thankful to the Yazd University for supporting this research.

\section{References}

Almalki, S. J. and Yuan, J. (2013). A new modified Weibull distribution. Reliability Engineering \& System Safety, 111:164-170.

Balakrishna, N. and Shiji, K. (2014). On a class of bivariate exponential distributions. Statistics \& Probability Letters, 85:153-160.

Barlow, R. E. (1968). Some recent developments in reliability theory. Technical report, Unirersity of California, Berkeley.

Basu, A. P. (1971). Bivariate failure rate. Journal of the American Statistical Association, 66(333):103-104.

Bebbington, M., Lai, C.-D., and Zitikis, R. (2007). A flexible Weibull extension. Reliability Engineering $\mathcal{E}$ System Safety, 92(6):719-726.

Burnham, K.P. and D.R. Anderson. (1998). Model selection and inference: a practical information-theoretic approach. Springer, New York.

Carrasco, J. M., Ortega, E. M., and Cordeiro, G. M. (2008). A generalized modified Weibull distribution for lifetime modeling. Computational Statistics \& Data Analysis, 53(2):450-462.

Chen, Z. (2000). A new two-parameter lifetime distribution with bathtub shape or increasing failure rate function. Statistics \& Probability Letters, 49(2):155-161.

Cordeiro, G. M., Ortega, E., and Silva, G. (2012). The beta extended Weibull family. Journal of Probability and Statistical Science, 10:15-40.

Cordeiro, G. M. and Silva, R. B. (2014). The complementary extended Weibull power series class of distributions. Ciência e Natura, 36(3):1-13.

Csörgö, S. and Welsh, A. (1989). Testing for exponential and Marshall-Olkin distributions. Journal of Statistical Planning and Inference, 23(3):287-300.

El-Bassioun, A. H., El-Damcese, M., Mustafa, A., and Eliwa, M. (2015). Bivariate exponentaited generalized Weibull-Gompertz distribution. arXiv preprint arXiv:1501.02241.
El-Gohary, A., Alshamrani, A., and Al-Otaibi, A. N. (2013). The generalized Gompertz distribution. Applied Mathematical Modelling, 37(1-2):13-24.

El-Gohary, A. and El-Morshedy, M. (2015). Bivariate exponentiated modified Weibull extension. arXiv preprint arXiv:1501.03528.

El-Sherpieny, E. A., Ibrahim, S. A., and Bedar, R. E. (2013). A new bivariate generalized Gompertz distribution. Asian Journal of Applied Sciences, 1(4):141-150.

Fisk, P. R. (1961). The graduation of income distributions. Econometrica, 29(2):171-185.

Fréchet, M. (1927). Sur la loi de probabilité de l'écart maximum. Annales de la societe Polonaise de Mathematique, 6:93-116.

Gompertz, B. (1825). On the nature of the function expressive of the law of human mortality, and on a new mode of determining the value of life contingencies. Philosophical Transactions of the Royal Society of London, 115:513-583.

Gupta, R. D. and Kundu, D. (1999). Generalized exponential distributions. Australian $\mathcal{E}$ New Zealand Journal of Statistics, 41(2):173-188.

Gurvich, M., Dibenedetto, A., and Ranade, S. (1997). A new statistical distribution for characterizing the random strength of brittle materials. Journal of Materials Science, 32(10):2559-2564.

Jamalizadeh, A. and Kundu, D. (2013). Weighted Marshall-Olkin bivariate exponential distribution. Statistics, 47(5):917-928.

Joe, H. (1997). Multivariate models and multivariate dependence concepts. CRC Press.

Johnson, N. L., Kotz, S., and Balakrishnan, N. (1995). Continuous Univariate Distributions, volume 1. John Wiley \& Sons, New York, second edition.

Kies, J. (1958). The strength of glass. Naval Research Laboratory, Washington D.C.

Kundu, D. and Gupta, R. D. (2009). Bivariate generalized exponential distribution. Journal of Multivariate Analysis, 100(4):581-593.

Kundu, D. and Gupta, R. D. (2010). Modified SarhanBalakrishnan singular bivariate distribution. Journal of Statistical Planning and Inference, 140(2):526-538.

Kundu, D. and Raqab, M. Z. (2005). Generalized Rayleigh distribution: different methods of estimations. Computational Statistics \& Data Analysis, 49(1):187-200. 
Lai, C., Xie, M., and Murthy, D. (2003). A modified Weibull distribution. IEEE Transactions on Reliability, 52(1):33-37.

Lomax, K. (1954). Business failures: Another example of the analysis of failure data. Journal of the American Statistical Association, 49(268):847-852.

Marshall, A. W. and Olkin, I. (1967). A multivariate exponential distribution. Journal of the American Statistical Association, 62(317):30-44.

McCool, J. I. (2012). Using the Weibull distribution: reliability, modeling and inference, volume 950. John Wiley \& Sons.

Meintanis, S. G. (2007). Test of fit for Marshall-Olkin distributions with applications. Journal of Statistical Planning and Inference, 137(12):3954-3963.

Mudholkar, G. S. and Srivastava, D. K. (1993). Exponentiated Weibull family for analyzing bathtub failure-rate data. IEEE Transactions on Reliability, 42(2):299-302.

Nadarajah, S. and Kotz, S. (2005). On some recent modifications of Weibull distribution. IEEE Transactions on Reliability, 54(4):561-562.

Nelson, R. B. (1999). An Introduction to Copulas. Springer, New York.

Nikulin, M. and Haghighi, F. (2006). A chi-squared test for power generalized Weibull family for the headand-neck cancer censored data. Journal of Mathematical Sciences, 133(3):1333-1341.

Pham, H. (2002). A vtub-shaped hazard rate function with applications to system safety. International Journal of Reliability and Applications, 3(1):1-16.

Pham, H. and Lai, C.-D. (2007). On recent generalizations of the Weibull distribution. IEEE Transactions on Reliability, 56(3):454-458.

Phani, K. K. (1987). A new modified Weibull distribution function. Journal of the American Ceramic Society, 70(8):182-184.

Rayleigh, L. (1880). On the resultant of a large number of vibrations of the same pitch and of arbitrary phase. Philosophical Magazine and Journal of Science, 10(60):7378.

Rodriguez, R. N. (1977). A guide to the Burr type XII distributions. Biometrika, 64(1):129-134.

Santos-Neto, M., Bourguignon, M., Zea, L. M., Nascimento, A. D., and Cordeiro, G. M. (2014). The Marshall-Olkin extended Weibull family of distributions. Journal of Statistical Distributions and Applications, 1(1):9.
Sarhan, A. M. and Apaloo, J. (2013). Exponentiated modified weibull extension distribution. Reliability Engineering E System Safety, 112:137-144.

Sarhan, A. M., Hamilton, D. C., Smith, B., and Kundu, D. (2011). The bivariate generalized linear failure rate distribution and its multivariate extension. Computational Statistics and Data Analysis, 55(1):644-654.

Sarhan, A. M. and Kundu, D. (2009). Generalized linear failure rate distribution. Communications in StatisticsTheory and Methods, 38(5):642-660.

Silva, R. B., Bourguignon, M., Dias, C. R. B., and Cordeiro, G. M. (2013). The compound class of extended Weibull power series distributions. Computational Statistics and Data Analysis, 58:352-367.

Smith, R. M. and Bain, L. J. (1975). An exponential power life-testing distribution. Communications in Statistics, 4(5):469-481.

Sullivan, J. and Joyce, P. (2005). Model selection in phylogenetics. Annual Review of Ecology, Evolution, and Systematics, 36:445-466.

Surles, J. G. and Padgett, W. J. (2001). Inference for reliability and stress-strength for a scaled Burr type $X$ distribution. Lifetime Data Analysis, 7(2):187-200.

Tahmasebi, S. and Jafari, A. A. (2015). Exponentiated extended Weibull-power series class of distributions. Ciência e Natura, 7(2):183-193.

White, J. S. (1969). The moments of log-Weibull order statistics. Technometrics, 11(2):373-386.

Xie, M. and Lai, C. D. (1995). Reliability analysis using an additive weibull model with bathtub-shaped failure rate function. Reliability Engineering $\mathcal{E}$ System Safety, 52(1):87-93.

Xie, M., Tang, Y., and Goh, T. N. (2002). A modified Weibull extension with bathtub-shaped failure rate function. Reliability Engineering \& System Safety, 76(3):279-285. 